\title{
Xerosis and pruritus as major EGFRI-associated adverse events
}

\author{
Julia M. K. Clabbers ${ }^{1}$ - Christine B. Boers-Doets ${ }^{1} \cdot$ Hans Gelderblom $^{1} \cdot$ Theo Stijnen $^{2}$ • \\ Mario E. Lacouture ${ }^{3}$ Koos J. M. van der Hoeven ${ }^{1}$ - Adrian A. Kaptein ${ }^{4}$
}

Received: 17 November 2014 / Accepted: 17 May 2015 / Published online: 27 June 2015

(C) The Author(s) 2015. This article is published with open access at Springerlink.com

\begin{abstract}
Purpose The objective of this sub-analysis of the BeCet study (NCT01136005) was to examine health-related quality of life (HRQoL) of patients experiencing dermatological adverse events (AEs) during the first 6 weeks of epidermal growth factor receptor inhibitor (EGFRI) treatment.

Methods Patients $(n=85)$ treated with EGFRI completed five questionnaires during the first 6 weeks of treatment. 77 patients provided enough data for the sub-analysis. Experienced AEs were reported in the Dermatological Reactions Targeted Therapy-Patients (DERETT-P), a symptom experience diary for patients treated with targeted therapy. The impact of EGFRI-associated dermatological adverse events on HRQoL was examined using four HRQoL questionnaires; the Functional Assessment of Cancer Therapy-EGFRI (FACTEGFRI-18), the Functional Assessment of Cancer TherapyGeneral (FACT-G), the 36-Item Short Form Health Survey (SF-36), and the Skindex-16.
\end{abstract}

Julia M. K. Clabbers and Christine B. Boers-Doets contributed equally to this work.

Julia M. K. Clabbers

juliamkclabbers@gmail.com

1 Department of Clinical Oncology, Leiden University Medical Centre, P.O. Box 9600, 2300 RC Leiden, The Netherlands

2 Department of Medical Statistics and Bioinformatics, Leiden University Medical Centre, P.O. Box 9600, 2300 RC Leiden, The Netherlands

3 Department of Medicine, Dermatology Service, Memorial Sloan-Kettering Cancer Center, New York, NY 10065, USA

4 Department of Medical Psychology, Leiden University Medical Centre, P.O. Box 9600, 2300 RC Leiden, The Netherlands
Results During the first 6 weeks of EGFRI treatment, physical discomfort was the most significantly affected domain. In the entire study population, xerosis (dry skin) $(22.3 \%)$ and pruritus (itchy skin) (16.9\%) were reported as the most impactful AEs. For patients experiencing a papulopustular eruption (acneiform rash) pruritus (24.2\%), xerosis (18.9\%), and papulopustular eruption $(6.3 \%)$ were reported as the most impactful AEs. Papulopustular eruption, xerosis, and pruritus all showed a significant negative effect on HRQoL, displayed in FACT-EGFRI-18 scores.

Conclusions In addition to papulopustular eruption, xerosis and pruritus are major EGFRI-associated dermatological AEs with an impact on HRQoL, which warrant more attention in clinical practice and research.

Keywords Cancer - Quality of life - Epidermal growth factor receptor inhibitor $\cdot$ Papulopustular eruption $\cdot$ Xerosis $\cdot$ Pruritus

\section{Background}

Epidermal growth factor receptor inhibitors (EGFRIs) are frequently used in treatment regimens of patients with solid tumors. Compared with cytotoxic chemotherapeutic agents, which may cause myelosuppression, nausea, vomiting, neuropathy, and alopecia, EGFRIs are associated with a lower incidence of systemic adverse events (AEs). However, patients treated with EGFRIs experience dermatological AEs (dAEs), such as papulopustular eruption (acneiform rash), xerosis (dry skin), pruritus (itchy skin), and paronychia (periungual inflammation), as well as mucosal and hair abnormalities [1,2].

The most common AE of EGFRI treatment is a papulopustular eruption, occurring in 75 to $95 \%$ of patients $[1,2]$. The papulopustular eruption consists of acneiform 
follicular and perifollicular papules and sterile pustules, most pronounced on the face, scalp, upper back, and chest and is often accompanied by xerosis and pruritus. The papulopustular eruption is a relatively early-onset $\mathrm{AE}$, usually occurring between 1 to 3 weeks after initiation of treatment. The incidence is higher with monoclonal antibodies like cetuximab and panitumumab (more than $88 \%$ ) than with tyrosine kinase inhibitors like gefitinib, erlotinib, lapatinib, and afatinib (43-75\%). In about 80 to $90 \%$ of the skin reactions, the worst recorded severity is mild (grade 1) to moderate (grade 2), but in $10 \%$ a more severe skin reaction (grade 3 ) is seen $[1,2]$. In several studies, the presence and severity of the eruption has shown a correlation with a positive response to cancer treatment, as expressed in higher median survival rates [1-3]. However, patients reported that the papulopustular eruption interferes in their daily activities and in the appearance of their skin, because the EGFRI-associated dAEs, often in visible areas, make them worried, frustrated, and depressed and cause withdrawal from social activities [3, 4].

The physical discomfort caused by EGFRI treatment has been identified as having the most impact on health-related quality of life (HRQoL), especially the sensations of pain, burning, and skin sensitivity. The dAEs may lead to a decreased HRQoL and to dose reduction or discontinuation of anticancer treatment, even though the treatment might be effective in treating the cancer and reducing the dose may negatively affect cancer outcome [4]. At present, the consequence of the dAEs on HRQoL in patients with cancer receiving EGFRI treatment remains poorly understood.

This sub-analysis of the ongoing BeCet study (NCT01136005) is aimed to provide a better understanding of HRQoL in patients with cancer receiving EGFRI treatment, using five different questionnaires.

\section{Patients and materials}

\section{Patients}

The study population was derived from the ongoing BeCet study. This phase III randomized double-blinded trial compares Bepanthen against cetomacrogol cream on their preventive effect in decreasing the incidence of grade $\geq 2$ EGFRIassociated papulopustular eruption and assesses the Dutch version of Functional Assessment of Cancer TherapyEGFRI (FACT-EGFRI-18) for reliability and validity [5, 6]. The study has been approved by the Medical Ethics Review Committees of each participating hospital. Twelve Dutch centers are currently recruiting patients starting with an EGFRI treatment for any type of cancer (i.e., panitumumab, cetuximab, lapatinib, gefitinib, erlotinib, or afatinib). Patients need to have an Eastern Co-operative Oncology Group performance status $\leq 2$ and need to be able to complete questionnaires.

The first 85 consecutive patients were included for this subanalysis between July 2010 and May 2014. This analysis studies the impact of the dAEs on the HRQoL, while the main study analyses the appearance and severity of dAEs. There are no strict criteria for the sample size in HRQoL studies. Within a homogenous population there is lower variability in answers on HRQoL items, making a smaller sample size acceptable.

\section{Materials}

During the 6-week study period, patients completed five different questionnaires. They completed the symptom experience diary Dermatological Reactions Targeted Therapy-Patients (DERETT-P) and the FACT-EGFRI-18 weekly. Within the BeCet trial, these two questionnaires are measured weekly to provide detailed information about the incidence and severity curve of dAEs. For this sub-analysis, fewer data than in the main study collected are of relevance. These are the data of weeks 0,2 , and 4 .

In week 4, the Functional Assessment of Cancer TherapyGeneral (FACT-G), the 36-Item Short Form Health Survey (SF-36), and the Skindex-16 questionnaires were completed for validation purposes of the FACT-EGFRI-18. Week 4 of treatment was chosen, because then most patients will have experienced a papulopustular eruption [7]. In this analysis, scores of these generic questionnaires were compared with previously published articles, to put the HRQoL of cancer patients treated with EGFRIs in perspective to similar samples.

\section{DERETT}

The Dermatological Reactions Targeted Therapy (DERETT) is available in two versions, for patients (DERETT-P) and healthcare professionals (DERETT-H), consisting of 61 and 50 items, respectively. These tools gather information such as area involved, severity and duration of the symptoms, products used to treat symptoms, effectiveness of the supportive care interventions, treatment adherence, and symptom-related distress [8]. DERETT provides a more precise and clinically relevant information on the patient's condition than Common Terminology Criteria for Adverse Events (CTCAE) grading alone.

\section{FACT-EGFRI-18}

The Functional Assessment of Cancer Therapy Questionnaire-EGFRI has been developed to assess HRQoL related to EGFRI-associated dAEs. The translation, linguistic validation, and qualitative assessment of the FACT-EGFRI-18 have been described $[5,6,9]$. The validations of the English and 
Dutch versions are ongoing. The FACT-EGFRI-18 consists of 18 items in three HRQoL domains: physical (7 items), social/ emotional (6 items), and functional (5 items). Scores are rated on a numerical analogue scale $(0=$ not at all, $4=$ very much). A high domain score reflects a low HRQoL. On the other hand, a high total score indicates a high HRQoL [10].

\section{FACT-G}

The FACT-G version 4 is a patient reposted outcome (PRO) measure with numerical analogue scales $(0=$ not at all, $4=$ very much). The FACT-G version 4 consists of 27 items in four HRQoL domains: physical (7 items), social/family (7 items), emotional (6 items), and functional well-being ( 7 items). High total scores indicate a high HRQoL. The FACT-G has been validated for patients with cancer in general [11].

$S F-36$

The 36-Item Short Form Health Survey (SF-36) is a generic HRQoL survey. The questionnaire consists of 36 items, covering eight scales: physical functioning, role limitations due to physical health and due to emotional problems, energy/fatigue, emotional well-being, social functioning, pain, and general health. A high total scale score represents a high HRQoL. The SF-36 can be used to measure HRQoL in general and specific populations, and has been validated for Dutch citizens, and patients with cancer [12].

\section{Skindex-16}

The Skindex-16 is a 16-item PRO assessing dermatological symptoms on a numerical analogue scale $(0=$ never bothered, $6=$ always bothered), where high scores represent a low HRQoL. It contains three domains: symptoms (4 items), emotions ( 7 items), and functioning (5 items). The Skindex-16 is reliable and valid for general skin diseases. It has been used more often to assess HRQoL in patients receiving EGFRI treatment, but does not address symptoms related to hair, nails, or mucous membranes, that are specific targets for EGFRIs [13].

\section{Statistical analysis}

Descriptive statistics were used to describe the study population. In DERETT-P, the incidences of the AEs with the highest impact on HRQoL (Fig. 1) were determined per week and in total. The domain and total scores of FACT-EGFRI-18 during the first 6 weeks of EGFRI treatment are displayed in a time plot (Fig. 2). With a one-way ANOVA, item and domain scores during treatment were compared to baseline, followed by a Bonferroni procedure to correct for multiple testing. Using the Mann-Whitney test, FACT-EGFRI-18 scores during week 2 to 4 and Skindex-16 scores of week 4 were compared between different subgroups, i.e., gender, type of cancer, EGFRI agent, and age, as mainly in those weeks papulopustular eruption manifests [1]. To analyze HRQoL for patients experiencing a papulopustular eruption, their FACT-EGFRI-18 scores were compared with pre-treatment scores, also using the Mann-Whitney test. With manual two-sample $t$ tests and one-sample Wilcoxon signed-rank tests, mean and median scores of FACT-G, SF-36, and Skindex-16 were both compared to scores in previously published articles, in order to determine how HRQoL relates to these populations. DERETT-P and FACT-EGFRI-18 scores have not been described before and could, therefore, not be compared. All data analysis was performed with the Statistical Package for Social Sciences (SPSS) version 20. Overall, $p<0.05$ was accepted as a statistically significant result.

\section{Results}

\section{Demographics}

Between July 2010 and May 2014, a total of 85 patients were included. Eight patients $(9.4 \%)$ with disease progression were excluded as they stopped EGFRI treatment before week 4 and, consequently, did not complete FACT-G, SF-36, and Skindex16. In total, 77 patients were evaluable. Six (7.79 \%) of them stopped EGFRI treatment after week 4 because of disease progression and/or death, but produced enough data to be considered evaluable for this study. The mean age of the included study population $(n=77)$ was 65.0 years (SD 9.91). Forty-six patients $(59.7 \%)$ were male. The majority of the patients were of Caucasian origin $(96.1 \%)$ and three patients of other origin (Asian and Hindu) (Table 1). Patients were mainly diagnosed with non-small cell lung cancer (NSCLC) and colorectal cancer, 41.6 and $39.0 \%$, respectively. Panitumumab $(37.7 \%)$ and erlotinib (32.5\%) were the most prescribed EGFRI drugs. Of the DERETT-P, FACT-EGFRI-18, FACT-G, SF-36, and Skindex-16 questionnaires, 50, 47.4, 25.9, 25.9, and $23.5 \%$, respectively, were not completed. The main reasons for the uncompleted questionnaires were that the healthcare provider did not hand out the questionnaire in the uneven weeks (weeks 1,3 , and 5) and early discontinuation due to disease progression. Some patients did not complete the questionnaires because they did not feel the need to do so since their AEs stayed almost the same as during the previous measures, most prominent in patients with many or nearly none experienced AEs. Patient burden and burn out may also play a role.

\section{Impact of various adverse events}

The DERETT-P questionnaire asks patients to report if they experienced certain AEs and in which severity. Secondly, the 
Table 1 Patient demographics $(n=77)$

\begin{tabular}{ll}
\hline Characteristic & Number of patients (\%) \\
\hline Gender & \\
Male & $46(59.7)$ \\
Female & $31(40.3)$ \\
Age in years & $65.0(9.91)[41-87]^{\mathrm{a}}$ \\
Race & \\
Caucasian & $74(96.1)$ \\
Other & $3(3.9)$ \\
Type of cancer & \\
NSCLC & $32(41.6)$ \\
Colorectal & $30(39.0)$ \\
HNC & $8(10.4)$ \\
Mamma & $3(3.9)$ \\
Pancreas & $3(3.9)$ \\
Osteosarcoma & $1(1.3)$ \\
EGFRI type & \\
Panitumumab & $29(37.7)$ \\
Erlotinib & $25(32.5)$ \\
Cetuximab & $10(13.0)$ \\
Gefitinib & $10(13.0)$ \\
Lapatinib & $3(3.9)$ \\
\hline
\end{tabular}

$S D$ standard deviation, NSCLC non-small cell lung cancer, $H N C$ head and neck cancer

${ }^{\text {a }}$ Expressed in mean (SD) [range]

questionnaire asks from which AE they experienced the most hinder. Xerosis and pruritus were reported most often: mean 22.3 and $16.9 \%$, respectively. The remaining dAEs were reported by means less than $4.8 \%$. Fig. 1a displays the incidence of the four AEs which have the highest impact on HRQoL during the 6-week study period, while Fig. 1b displays the AEs with the highest impact on HRQoL over time. The peak of impact of xerosis on HRQoL was in week 5 $(33.3 \%)$, and at week 6 for pruritus $(25.0 \%)$. Papulopustular eruption was reported as having the most impact on HRQoL by $4.2 \%$ of all patients, with a peak in week $4(9.4 \%)$.

Since a papulopustular eruption may overlap xerosis and pruritus and, therefore, the outcome may be different in patients who did develop a papulopustular eruption compared to those who did not, we explored the patients that experienced a papulopustular eruption separately. Even in this subgroup, AEs having most impact on HRQoL remained pruritus $(24.2 \%)$, xerosis $(18.9 \%)$, a burning sensation of the skin $(8.4 \%)$, and lastly a papulopustular eruption (6.3\%).

\section{Quality of life during EGFRI treatment}

Table 2 and Fig. 2 show the development of total and domain scores of FACT-EGFRI-18 over time. Scores on the physical domain were significantly higher during all 6 weeks compared to baseline $(p<0.001)$. The functional domain for all grades showed a significantly higher score in the sixth week compared to baseline $(p=0.039)$. In patients with grade $1 / 2$, the dispersion in these domains is relatively low (Fig. 2c). However, the social-emotional domain did show significant changes within the grade $3 / 4$ sample (Fig. 2d). What stands out is the large spread on the domains of "social-emotional" and "functional" in patients with grade $3 / 4$ at weeks 0 and 1 . This was also the case prior to the start of the EGFRI treatment; in week 0 , the standard error is negative and as the weeks pass this spread decreases. For all domains and items, a higher score represents lower HRQoL. The total FACT-EGFRI-18 score decreased during treatment, reflecting decrease in HRQoL.

There were no significant differences between FACTEGFRI-18 scores for gender (total score men 63.40, women 63.92) or cancer type (total scores ranging from 63.7 to $68.00)$. Patients younger than 50 years scored significantly $(p=0.015)$ lower on the functional domain (score 0.91 $<50$ years versus $1.6161-70$ years (mean age)). Patients above 81 years experienced more impact on the physical domain $(p=0.028)$ (2.94 versus 5.06 in the mean age group of $61-70)$ and total score $(p=0.020)(68.56$ by $>81 ; 63.84$ by $61-$ $70)$, compared to patients in the mean age range between 61 and 70 years.

The presence of papulopustular eruption during the study period significantly decreased HRQoL as measured by FACTEGFRI-18 $(p<0.001)$. This was most prominent for the physical domain (Table 3). FACT-EGFRI-18 scores were also analyzed separately for xerosis and pruritus, showing a significant reduced HRQoL $(p<0.014)$.

FACT-G scores were compared with the scores of Cella et al. [11], which included other types of cancer, i.e., leukemia, lymphoma, prostate cancer, and ovarian cancer without EGFRI treatment. Our study population scored significantly higher on the physical ( $p=0.014)$ and emotional domains $(p=0.013)$, with higher scores indicating a higher HRQoL. Scores on the socialfamily and functional domains did not differ significantly.

Scores on SF-36 were first compared to scores of a sample of a Dutch healthy control population in order to examine the difference in HRQoL of EGFRI treated cancer patients with healthy individuals [14]. The current study population had a higher mean age and scored significantly lower on all domains $(p<0.028)$, meaning lower HRQoL. Secondly, SF-36 scores were compared with a group of cancer patients about to start chemotherapy or radiotherapy. The current study population was older, consisted of fewer females, had more patients with NSCLC and colorectal cancer, and fewer with breast cancer. Scores were similar for most SF-36 domains. Only for physical functioning $(p=0.042)$ and general health $(p<0.001)$, the current study population scored significantly lower, meaning a lower HRQoL [14].

Skindex-16 separately identified a significant lower total score when papulopustular eruption was present $(p<0.002)$, 
a

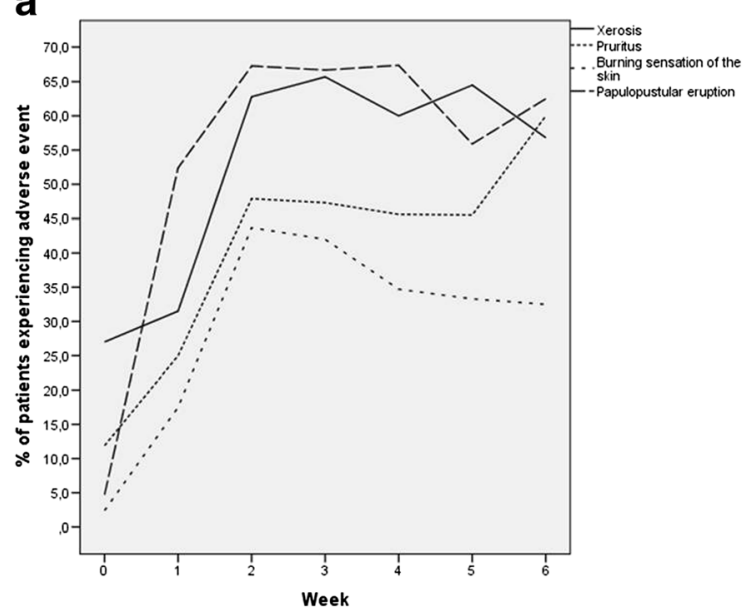

Fig 1 a Adverse events that patients reported as present in DERETT-P compared to $\mathbf{b}$ adverse events as having most impact on HRQoL as measured by DERETT-P. In (a), papulopustular eruption is reported as

but not for the presence of xerosis or pruritus. The Skindex-16 scores did not differ significantly between patients experiencing papulopustular eruption, xerosis, or pruritus (Table 4).

The current Skindex-16 scores were compared with the data of Joshi et al. [4] and Rosen et al. [15], both a retrospective investigation of Skindex-16 scores of patients with dAEs due to cancer treatment in a specialty referral clinic. The study of Joshi et al. is the most comparable to the current study as they focused on patients treated with EGFRIs. Joshi et al. [4] analyzed more women, more patients younger than 50 years, and more patients treated with cetuximab and erlotinib. Rosen et al. [15] included patients with targeted as well as non-

Table 2 Domain and total scores FACT-EGFRI-18 per week

\begin{tabular}{lllll}
\hline Week & \multicolumn{2}{l}{ Domain scores } & Total score \\
\cline { 2 - 4 } & $\begin{array}{l}\text { Physical } \\
7 \text { items }\end{array}$ & $\begin{array}{l}\text { Social-emotional } \\
6 \text { items }\end{array}$ & $\begin{array}{l}\text { Functional } \\
5 \text { items }\end{array}$ \\
\hline 0 & $1.41(1.80)$ & $0.490(2.20)$ & $0.470(1.69)$ & $69.5(5.23)$ \\
1 & $2.90(3.49)$ & $1.29(2.80)$ & $0.760(2.21)$ & $67.0(8.03)$ \\
2 & $5.42(4.13)$ & $1.45(2.01)$ & $1.200(1.86)$ & $63.8(7.00)$ \\
3 & $5.32(4.10)$ & $1.08(2.10)$ & $0.970(1.83)$ & $64.6(6.95)$ \\
4 & $6.00(5.14)$ & $1.57(2.60)$ & $1.780(3.27)$ & $62.6(9.75)$ \\
5 & $5.41(4.24)$ & $1.86(3.02)$ & $1.410(2.69)$ & $63.2(8.97)$ \\
6 & $6.65(5.01)$ & $1.70(3.28)$ & $1.870(3.13)$ & $61.6(10.1)$ \\
\hline
\end{tabular}

Scores are presented in mean (standard deviation). Domain scores are calculated as the sum of all corresponding items, taking into account that at least $50 \%$ of the items need to be answered for a reliable calculation. The total score is calculated by subtracting the domain scores from 72 (the maximum possible total score) and correct for the number of answered items. Therefore, a low HRQoL is reflected by a high domain score and a low total score

FACT-EGFRI-18 Functional Assessment of Cancer Therapy-EGFRI

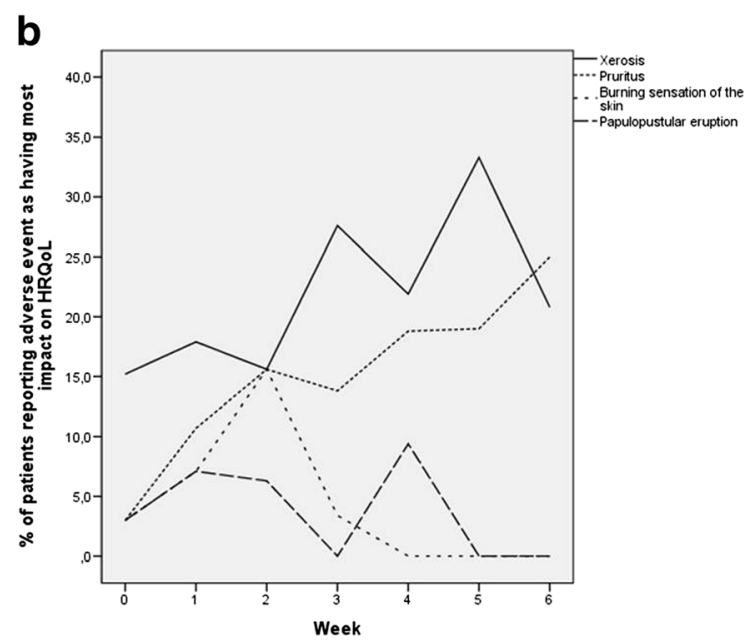

the most common adverse event, while $(b)$ displays that xerosis and pruritus have a more profound impact on HRQoL. HRQoL healthrelated quality of life

targeted therapy, who were generally younger, more often female, and less often of Caucasian ethnicity. Our study patients with papulopustular eruption and xerosis scored higher HRQoL on all Skindex-16 domains as patients in Joshi et al. [4] $(p<0.001)$ and in Rosen et al. [15] $(p<0.032)$. This was most marked on the emotional level. Our patients with pruritus had equal scores on the physical domain compared to both studies, and a comparable score on the functional domain with patients in Rosen et al. [15] receiving non-targeted therapy (Table 4). Even though not significant in the relatively small sample size, patients with pruritus showed a trend of higher scores on Skindex-16 and FACT-EGFRI-18 (indicating a lower HRQoL) than patients with papulopustular eruption or xerosis.

\section{Discussion}

The current results show that xerosis and pruritus have a major negative impact on HRQoL during the first 6 weeks of EGFRI treatment. This also applies for the patients affected by papulopustular eruption, from which only $6.3 \%$ report the presence of papulopustular eruption as having the highest impact on HRQoL. These findings were confirmed also in the STEPP trial $[16,17]$.

In Gandhi et al. [18], patients reported xerosis as having the most negative impact on HRQoL and pruritus as the third most impactful of all dAEs. In addition, xerosis was reported as having the second most negative impact on HRQoL of all unexpected AEs due to cancer treatment. Since xerosis and pruritus are less frequent reported EGFRI-associated dAEs, not all patients are counseled about these possible dAEs before initiating treatment. Therefore, they cannot engage in 
a
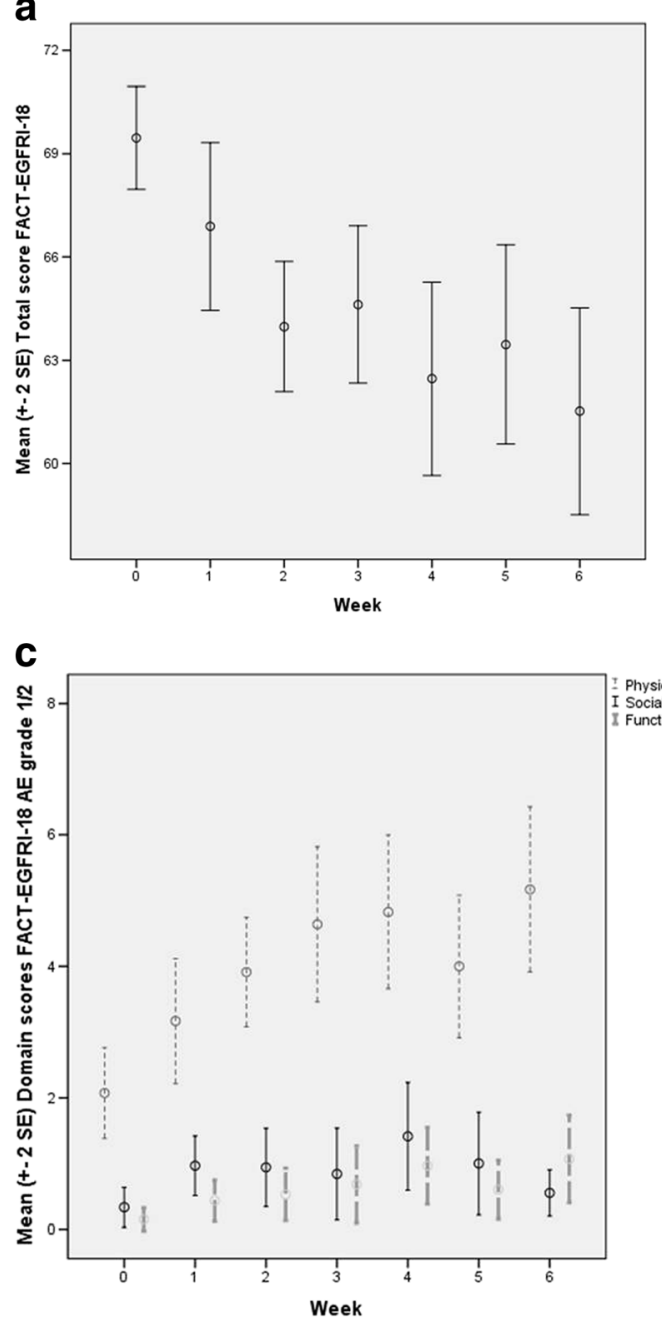

Fig. 2 a Mean (standard error of the mean) FACT-EGFRI-18 total scores per week. b Mean (standard error of the mean) of all grade FACT-EGFRI18 domain scores per week. c Mean (standard error of the mean) of grade 1/2 FACT-EGFRI-18 domain scores per week. d Mean (standard error of b

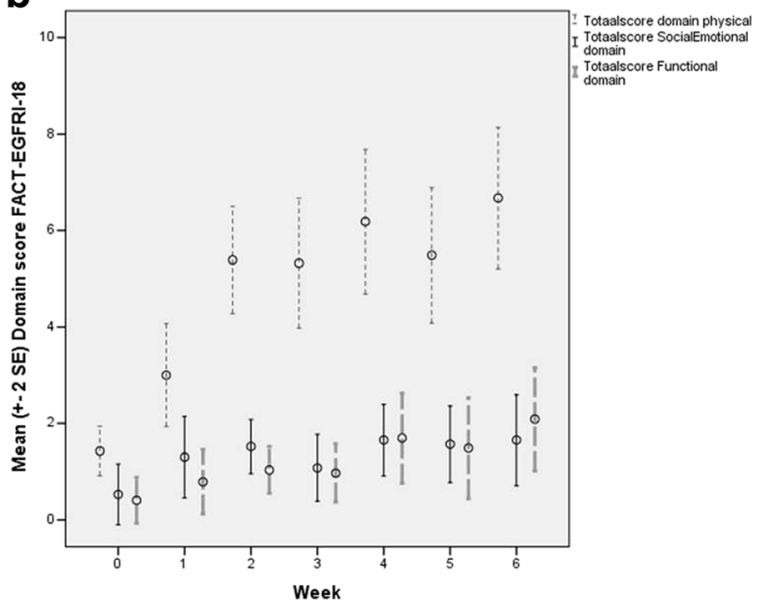

d

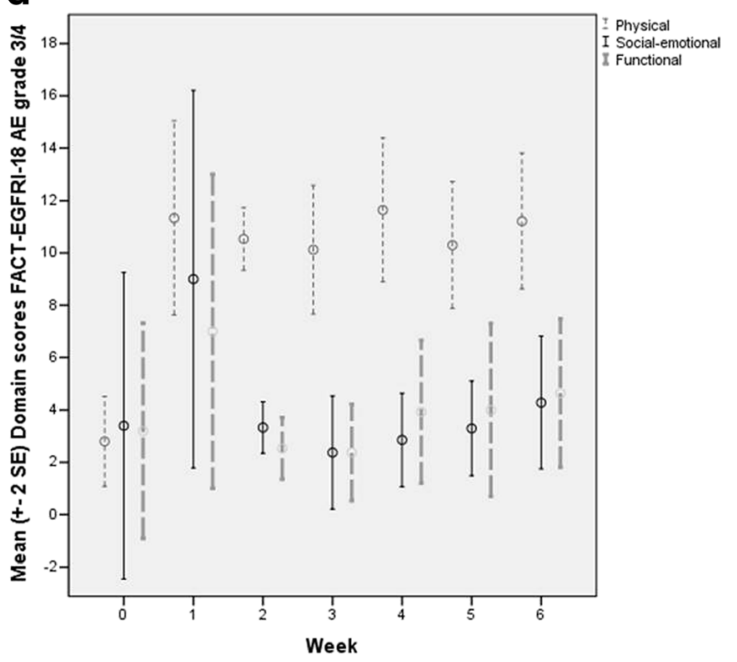

the mean) of grade 3/4 FACT-EGFRI-18 domain scores per week. FACTEGFRI-18 Functional Assessment of Cancer Therapy-EGFRI, EGFRI epidermal growth factor receptor inhibitor

Table 3 HRQoL with papulopustular eruption displayed in FACT-EGFRI-18 scores

\begin{tabular}{lllll}
\hline \multirow{2}{*}{ Week } & Papulopustular eruption & \multicolumn{2}{l}{ Domain scores FACT-EGFRI-18 } \\
\cline { 3 - 5 } & & Physical & Social-emotional & Functional \\
\hline 0 & $n=0$ & $1.41(1.80)$ & $0.490(2.20)$ & $0.470(1.69)$ \\
1 & $n=19$ & $4.58(4.03)^{*}$ & $2.21(3.65)^{*}$ & $1.42(3.06)^{*}$ \\
2 & $n=31$ & $6.97(3.70)^{*}$ & $2.00(2.21)^{*}$ & $1.61(1.94)^{*}$ \\
3 & $n=24$ & $6.54(3.98)^{*}$ & $1.50(2.45)^{*}$ & $1.77(2.85)^{*}$ \\
4 & $n=30$ & $7.27(5.75)^{*}$ & $2.15)^{*}$ \\
5 & $n=19$ & $6.79(4.13)^{*}$ & $1.92(2.80)^{*}$ & $1.84(3.08)^{*}$ \\
6 & $n=24$ & $7.13(4.16)^{*}$ & $1.92(2.21)^{*}$ \\
\hline
\end{tabular}

Scores presented in mean (SD)

FACT-EGFRI-18 Functional Assessment of Cancer Therapy-EGFRI, HRQoL Health-related quality of life ${ }^{*} p<0.05$, a significantly lower HRQoL compared with week 0 (baseline) 
Table 4 Comparison of mean and median Skindex-16 scores for patients experiencing dermatological adverse events

\begin{tabular}{|c|c|c|c|c|c|c|}
\hline Skindex-16 domains & $\begin{array}{l}\text { Current study } \\
\text { Papulopustular eruption } \\
n=30\end{array}$ & $\begin{array}{l}\text { Current study } \\
\text { Xerosis } \\
n=24\end{array}$ & $\begin{array}{l}\text { Current study } \\
\text { Pruritus } \\
n=21\end{array}$ & $\begin{array}{l}\text { Joshi et al. }{ }^{4} \\
\text { EGFRI } \\
\text { AEs } \\
n=67\end{array}$ & $\begin{array}{l}\text { Rosen et al. }{ }^{14} \\
\text { Targeted therapy AEs } \\
n=163\end{array}$ & $\begin{array}{l}\text { Rosen et al. }{ }^{14} \\
\text { Non-targeted therapy AEs } \\
n=120\end{array}$ \\
\hline \multicolumn{7}{|l|}{ Symptoms } \\
\hline Mean (SD) & $27.2(26.4)$ & $22.9(26.5)$ & $35.6(28.9)$ & $45.3^{\mathrm{a}, \mathrm{b}, \mathrm{c}}$ & & \\
\hline Median $(95 \% \mathrm{CI})$ & $20.8(12.5-33.3)$ & $16.7(0.00-29.2)$ & $29.2(20.8-37.5)$ & & $37.5(29.2-54.2)^{\mathrm{b}, \mathrm{c}}$ & $39.6(25.0-45.8)^{\mathrm{b}, \mathrm{c}}$ \\
\hline \multicolumn{7}{|l|}{ Emotions } \\
\hline Mean (SD) & $17.2(21.9)$ & $14.7(19.3)$ & $21.4(24.2)$ & $50.0^{\mathrm{a}, \mathrm{b}, \mathrm{c}, \mathrm{d}}$ & & \\
\hline Median $(95 \% \mathrm{CI})$ & $9.53(4.77-21.4)$ & $9.53(0.00-21.4)$ & $14.3(4.77-26.2)$ & & $50.0(40.5-57.1)^{\mathrm{b}, \mathrm{c}, \mathrm{d}}$ & $38.1(30.4-47.6)^{\mathrm{b}, \mathrm{c}, \mathrm{d}}$ \\
\hline \multicolumn{7}{|l|}{ Functioning } \\
\hline Mean (SD) & $10.0(17.1)$ & $7.23(14.3)$ & $10.5(15.7)$ & $31.3^{\mathrm{a}, \mathrm{b}, \mathrm{c}, \mathrm{d}}$ & & \\
\hline Median $(95 \% \mathrm{CI})$ & $0.00(0.00-10.2)$ & $0.00(0.00-3.34)$ & $3.34(0.00-16.7)$ & & $16.7(8.2-26.7)^{\mathrm{b}, \mathrm{c}, \mathrm{d}}$ & $13.3(3.3-20.0)^{\mathrm{b}, \mathrm{c}}$ \\
\hline
\end{tabular}

Joshi et al. [4] displayed mean scores while Rosen et al. [14] displayed median scores. Therefore, in the current study, both are displayed to make comparison possible

$A E$ adverse event

${ }^{a}$ No standard deviations were given in Joshi et al. [4]

${ }^{\mathrm{b}}$ A statistically significant result compared with patients from the current study experiencing papulopustular eruption

${ }^{\mathrm{c}}$ A statistically significant result compared with patients from the current study experiencing xerosis

${ }^{\mathrm{d}}$ A statistically significant result compared with patients from the current study experiencing pruritus

anticipatory coping; a method to deal with anticipated AEs. In an interview study of Frith et al. [19], strategies were identified for patients to cope with anticipated cancer treatment AEs. First, patients try to foresee the amount of distress and accompanying emotions through "affective rehearsal," followed by acceptance of possible AEs and gathering resources to manage them through "behavioral rehearsal," a method to modify interpersonal skills and social interactions. The final strategy is finding ways to control the development of the AEs and the personal emotional reactions on them [19].

Since this is the first report of FACT-EGFRI-18 scores, we are not able to compare our data to data from other trials. Our analysis showed that during the first 6 weeks of EGFRI treatment, patients experience influence on their HRQoL primarily due to physical symptoms, especially irritation, xerosis, pruritus, and nail sensitivity. The reversed FACT-EGFRI-18 scores of papulopustular eruption, xerosis and pruritus decreased significantly on the total scores $(p<0.014)$ indicating a high HRQoL. The non-reversed FACT-EGFRI-18 scores increased significantly on the domain scores $(p<0.012)$ reflecting a low HRQoL. Only patients experiencing xerosis in week 1 and patients experiencing pruritus in week 5 did not have a significant higher score on the functional domain, meaning a non-significant different score compared to before treatment.

Cella et al. [11] used the FACT-G questionnaire in a population with different cancer types and treatments, and EGFRI-treated patients were not included. EGFRI treatment is considered more tolerable compared to conventional cytotoxic treatments, because systemic AEs are less frequent [20]. This could explain the higher score on the physical and emotional domains in the current study population. Aaronson et al. [14] measured pre-treatment SF-36 scores, resulting in fewer AEs, which explain a higher physical functioning and general health.

The study of Joshi et al. [4] analyzed Skindex-16 scores at any time of AE development instead of the current fixed measurement at week 4 of treatment. In addition, the referral to a specialty clinic might have increased patients' worry about the severity of the AEs. Rosen et al. [15] measured patients at any moment during all types of cancer treatment, causing a broader range of dAEs, which influences Skindex-16 scores. This could also explain a better HRQoL for patients with papulopustular eruption or xerosis. In addition, patients with pruritus had similar Skindex-16 scores on the physical domain as the patients in the specialty referral clinic in Joshi et al. [4] and Rosen et al. [15]. These findings suggest that the impact of pruritus on HRQoL might be larger than papulopustular eruption and xerosis. The similar score on the physical domain of Skindex-16 of patients with acne vulgaris suggests clinical similarities with EGFRI papulopustular eruptions. Patients with EGFRI-associated papulopustular eruption are generally more likely to accept the temporary eruption as part of their treatment for cancer, especially since they are usually informed about its association with effectiveness of treatment, which can clarify the different impact on emotions and functioning $[17,20,21]$. 
This study required a substantial effort for patients to complete consecutive questionnaires at the intended assessments. There might be a selection bias as missing data were from relatively sicker patients, which could result in overestimating the overall HRQoL and underestimating the impact of dAEs on HRQoL. Another cause may be the missing data from patients who did not experience noticeable $\mathrm{AE}$ changes and, therefore, did not complete the questionnaires in the weeks without $\mathrm{AE}$ changes. The incidence of dAEs might be reduced as all patients received close monitoring and preventive and reactive treatment. As all factors mentioned above are more likely to have improved HRQoL of patients, the expectation is that the current results are indeed realistic and may be even more profound when less confounding factors would be present. Because the study population consisted mainly of patients from Caucasian origin, with NSCLC or colorectal cancer, current results may not apply to all EGFRI-treated cancer patients.

\section{Conclusion}

Clinical and research endeavors in patients with various cancers who receive medical management consisting of EGFRIs have focused mainly on papulopustular eruption as an EGFRI-associated AE, which resulted in an important decrease in HRQoL. However, the current study shows that xerosis and pruritus are also important AEs with a major impact on HRQoL. This justifies more focus on HRQoL related to these symptoms and on their prevention and treatment in future research.

In clinical practice, xerosis and pruritus are infrequently discussed during patient counseling prior to treatment, as they are less visible than the more common papulopustular eruption. Providing patients adequate information about treatment and possible AEs has shown a positive result on patients' emotional and physical well-being. Counseling patients prior to EGFRI treatment about potential xerosis and pruritus is therefore important, as well as taking preventive measures against these AEs [18, 19, 22, 23].

Conflict of interest MEL has consulted for Roche, BMS, BI, Genentech, AZ, and Merck. The other authors have no conflict of interest to declare in relation to this work.

Open Access This article is distributed under the terms of the Creative Commons Attribution-NonCommercial 4.0 International License (http:// creativecommons.org/licenses/by-nc/4.0/), which permits any noncommercial use, distribution, and reproduction in any medium, provided you give appropriate credit to the original author(s) and the source, provide a link to the Creative Commons license, and indicate if changes were made.

\section{References}

1. Lacouture ME, Anadkat MJ, Bensadoun RJ, Bryce J, Chan A, Epstein JB, Eaby-Sandy B, Murphy BA (2011) Clinical practice guidelines for the prevention and treatment of EGFR inhibitorassociated dermatologic toxicities. Support Care Cancer 19(8): 1079-1095. doi:10.1007/s00520-011-1197-6

2. Abdullah SE, Haigentz M, Jr., Piperdi B (2012) Dermatologic toxicities from monoclonal antibodies and tyrosine kinase inhibitors against EGFR: pathophysiology and management. Chemother Res Pract 2012:351210.doi: $10.1155 / 2012 / 351210$

3. Wagner LI, Lacouture ME (2007) Dermatologic toxicities associated with EGFR inhibitors: the clinical psychologist's perspective. Impact on health-related quality of life and implications for clinical management of psychological sequelae. Oncology (Williston Park) 21(11 Suppl 5):34-36

4. Joshi SS, Ortiz S, Witherspoon JN, Rademaker A, West DP, Anderson R, Rosenbaum SE, Lacouture ME (2010) Effects of epidermal growth factor receptor inhibitor-induced dermatologic toxicities on quality of life. Cancer 116(16):3916-3923. doi:10.1002/ cncr.25090

5. Boers-Doets CB, Gelderblom H, Lacouture ME, Bredle JM, Epstein JB, Schrama NA, Gall H, Ouwerkerk J, Brakenhoff JA, Nortier JW, Kaptein AA (2013) Translation and linguistic validation of the FACT-EGFRI-18 quality of life instrument from English into Dutch. Eur J Oncol Nurs 17(6):802-807. doi:10.1016/j.ejon. 2013.03.004

6. Boers-Doets CB, Gelderblom H, Lacouture ME, Epstein JB, Nortier JW, Kaptein AA (2013) Experiences with the FACTEGFRI-18 instrument in EGFRI-associated mucocutaneous adverse events. Support Care Cancer 21(7):1919-1926

7. Scope A, Agero AL, Dusza SW, Myskowski PL, Lieb JA, Saltz L, Kemeny NE, Halpern AC (2007) Randomized double-blind trial of prophylactic oral minocycline and topical tazarotene for cetuximabassociated acne-like eruption. J Clin Oncol 25(34):5390-5396

8. Boers-Doets CB (2014) The Target System - approach to assessment, grading, and management of dermatological \& mucosal side effects of targeted anticancer therapies. 1 ed. Wormer: IMPAQTT

9. Wagner LI, Lai SE, Aneja M, LoRusso P, Perez-Soler R, O’Brien B et al. (2007) Development of a functional assessment of side-effects to therapy (FAST) questionnaire to assess dermatology-related quality of life in patients treated with EGFR inhibitors (EGFRI): the FAST-EGFRI. ASCO, Meeting Abstracts 25[18 Suppl], 19532.

10. Wagner LI, Berg SR, Gandhi M, Hlubocky FJ, Webster K, Aneja M, Cella D, Lacouture ME (2013) The development of a Functional Assessment of Cancer Therapy (FACT) questionnaire to assess dermatologic symptoms associated with epidermal growth factor receptor inhibitors (FACT-EGFRI-18). Support Care Cancer 21(4): 1033-1041. doi:10.1007/s00520-012-1623-4

11. Cella DF, Tulsky DS, Gray G, Sarafian B, Linn E, Bonomi A, Silberman M, Yellen SB, Winicour P, Brannon J (1993) The Functional Assessment of Cancer Therapy scale: development and validation of the general measure. J Clin Oncol 11(3):570-579

12. Newnham EA, Harwood KE, Page AC (2007) Evaluating the clinical significance of responses by psychiatric inpatients to the mental health subscales of the SF-36. J Affect Disord 98(1-2):91-97. doi: 10.1016/j.jad.2006.07.001

13. Chren MM, Lasek RJ, Sahay AP, Sands LP (2001) Measurement properties of Skindex-16: a brief quality-of-life measure for patients with skin diseases. J Cutan Med Surg 5(2):105-110. doi:10.1007/ s102270000010

14. Aaronson NK, Muller M, Cohen PD, Essink-Bot ML, Fekkes M, Sanderman R, Sprangers MA, Velde AtT, Verrips E (1998) Translation, validation, and norming of the Dutch language version 
of the SF-36 Health Survey in community and chronic disease populations. J Clin Epidemiol 51(11):1055-1068

15. Rosen AC, Case EC, Dusza SW, Balagula Y, Gordon J, West DP, Lacouture ME (2013) Impact of dermatologic adverse events on quality of life in 283 cancer patients: a questionnaire study in a dermatology referral clinic. Am J Clin Dermatol 14(4):327-333. doi:10.1007/s40257-013-0021-0

16. Lacouture ME, Mitchell EP, Piperdi B, Pillai MV, Shearer H, Iannotti N, Xu F, Yassine M (2010) Skin toxicity evaluation protocol with panitumumab (STEPP), a phase II, open-label, randomized trial evaluating the impact of a pre-emptive skin treatment regimen on skin toxicities and quality of life in patients with metastatic colorectal cancer. J Clin Oncol 28(8):1351-1357. doi:10.1200/ JCO.2008.21.7828

17. Coleman S, Jatoi A (2010) STEPP for the EGFR inhibitor-induced rash - definitely a step in the right direction. Curr Oncol Rep 12(4): 223-225. doi:10.1007/s11912-010-0102-7

18. Gandhi M, Oishi K, Zubal B, Lacouture ME (2010) Unanticipated toxicities from anticancer therapies: survivors' perspectives. Support Care Cancer 18(11):1461-1468. doi:10.1007/s00520009-0769-1
19. Frith H, Harcourt D, Fussell A (2007) Anticipating an altered appearance: women undergoing chemotherapy treatment for breast cancer. Eur J Oncol Nurs 11(5):385-391. doi:10.1016/j.ejon.2007. 03.002

20. White KJ, Roydhouse JK, Scott K (2011) Psychosocial impact of cutaneous toxicities associated with epidermal growth factor receptor-inhibitor treatment. Clin J Oncol Nurs 15(1):88-96. doi: 10.1188/11.CJON.88-96

21. Balagula Y, Garbe C, Myskowski PL, Hauschild A, Rapoport BL, Boers-Doets CB, Lacouture ME (2011) Clinical presentation and management of dermatological toxicities of epidermal growth factor receptor inhibitors. Int J Dermatol 50(2):129-146. doi:10.1111/j. 1365-4632.2010.04791.x

22. Haley AC, Calahan C, Gandhi M, West DP, Rademaker A, Lacouture ME (2011) Skin care management in cancer patients: an evaluation of quality of life and tolerability. Support Care Cancer 19(4):545-554. doi:10.1007/s00520-010-0851-8

23. Siminoff LA (1992) Improving communication with cancer patients. Oncology (Williston Park) 6(10):83-87 